ten uns zu der Hypothese, daß die Synthese von ribosomaler RNS und messenger-RNS in Säugetierzellen unabhängig voneinander reguliert werden ${ }^{12}$. Für diese Hypothese sprechen auch die in der folgenden Arbeit mitgeteilten Befunde, die zeigen, daß durch Actinomycin in geringen Konzentrationen die Synthese der ribosomalen RNS vollständig unterbunden wird, während die Synthese der messengerRNS noch weiterläuft.

Die Speicherung von hochmolekularer messengerRNS in Zellen unter ungünstigen Bedingungen könnte durch einen Versuch der Zellen bedingt sein,

12 G. Косн u. H. Kubinski, 6th Intern. Congr. Biochem. New York, 1964. durch Synthese von spezifischen RNS-Arten den Wachstumsblock zu überwinden. Die RNS-Akkumulierung könnte aber auch durch Ausnutzung noch verfügbarer Synthesekapazitäten für RNS zustandekommen, und die synthetisierte RNS könnte bei Überführung der Zellen in bessere Wachstumsbedingungen dann ihre biologische Aktivität entfalten. In weiteren Untersuchungen wird versucht, darüber Auskunft zu erhalten.

Frau M. Paul und Frl. I. Vollertsen danken wir für gewissenhafte technische Assistenz. Die Arbeit wurde durchgeführt mit Unterstützung des Bundes ministeriums für wissenschaftliche Forschung und der Deutschen For. $\mathrm{schungsgemeinschaft.}$

\title{
Versuche zur Anreicherung von Hefe-Mangelmutanten, insbesondere mit Penicillin
}

\author{
O. Oltmanns und F. Lingens
}

Biochemische Abteilung des Chemischen Institutes der Universität Tübingen

(Z. Naturforschg. 21 b, 266—273 [1966] ; eingegangen am 30. September 1965)

\begin{abstract}
Bei der Suche nach einem Anreicherungsverfahren für biochemische Mangelmutanten von Hefen wurden mehrere Agenzien ausgetestet. Nur mit Penicillin konnten Anreicherungseffekte erzielt werden. Der Anteil der Mutanten stieg durch die Penicillinbehandlung von $10 \%$ nach der Methansulfonsäureäthylester-Induktion auf 20-50 Prozent. Veränderungen in der Häufigkeit der Mutantentypen mindern den Nutzeffekt des Anreicherungsverfahrens.
\end{abstract}

Biochemische Mangelmutanten werden für genetische und biochemische Untersuchungen in großer Zahl benötigt. Um den Arbeitsaufwand zu ihrer Isolierung klein zu halten, bedient man sich möglichst hochwirksamer Mutagene, oder man reichert die durch Mutagene induzierten Mutanten in einem besonderen Verfahren an. Bei Bakterien wird meistens das Verfahren von Lederberg und Zinder ${ }^{1}$ und Davis ${ }^{2}$ verwendet, bei dem die Wildstammzellen in Minimalmedium selektiv durch Pencillin abgetötet werden, während die Mutanten am Leben bleiben. Schimmelpilz-Mutanten lassen sich durch die SporenFiltrationstechnik (z. B. für Neurospora ${ }^{3}$ ) und durch Inosit-Verarmung Inosit-bedürftiger Stämme ${ }^{4}$ anreichern. Mit letzterer Methode (,,inositless

1 J. Lederberg u. N. Zinder, J. Amer. chem. Soc. 70, 4267 [1948].

2 B. D. Davis, J. Amer. chem. Soc. 70, 4267 [1948].

3 V. W. Woodward, J. R. DeZeeuw u. A. M. SRb, Proc. nat. Acad. Sci. USA 40, 192 [1954].

${ }^{4}$ H. E. Lester u. S. R. Gross, Science [Washington] 129, 572 [1959]. death") hat kürzlich Megnet ${ }^{5}$ auch bei Schizosaccharomyces pombe Erfolg gehabt. Für Saccharomyces konnte noch kein Anreicherungsverfahren entwickelt werden, obwohl es an Versuchen dazu nicht gefehlt hat ${ }^{6}$.

Wir hatten in einer früheren Arbeit die Wirksamkeit verschiedener Mutagene bei Saccharomyces geprüft und Mutationsraten von etwa $4-5 \%$ gefunden ${ }^{7}$. Das Ziel der hier zu beschreibenden Untersuchungen war es, eine weitere Steigerung der Mutantenausbeute durch Anwendung von Anreicherungsverfahren zu erreichen. Es lag nahe, entspresprechend der Penicillin-Methode Pilzantibiotika an den Hefen auszutesten. Diese Versuche führten jedoch schon früher nicht zu dem gewünschten Er-

5 R. Megnet, Experientia [Basel] 20, 320 [1964].

6 A. G. Moat, N. Peters, jr. u. A. M. Srb, J. Bacteriol. 77. 673 [1959].

7 F. Lingens u. O. Oltmanss, Z. Naturforschg. 19 b, 1058 [1964]. 
folg ${ }^{6}$, und auch wir konnten, wie gezeigt werden soll, keine Anreicherung nach Einwirkung von Pilzantibiotika und einigen anderen Stoffen beobachten. Dagegen schienen Versuche mit Penicillin überraschenderweise auch bei Hefen erfolgversprechend zu $\operatorname{sein}^{8}$.

\section{Methodik}

Wir verwendeten für alle Versuche den haploiden, wenig klumpenden Stamm S $288 \mathrm{C}$ von Sacch. cerevisiae, den wir Herrn Dr. R. K. Mortimer, Berkeley, Kalifornien, verdanken und einige daraus gewonnene Mangelmutanten.

Dieser Stamm enthielt in früheren Versuchen vermutlich auch diploide Zellen. Fräulein Dr. I. MüLler, Institut für Entwicklungsphysiologie der Universität Köln, konnte in unserer Kultur diploide neben haploiden Zellen nachweisen, die beide gleich schnell wachsen. Wir danken Fräulein Dr. I. Müller für die Isolierung eines nicht sporulierenden, allem Anschein nach rein haploiden Klones. Es läßt sich nicht mehr feststellen, wann die Teildiploidisierung unserer Kultur eingetreten ist. Vermutlich durch Verwendung des rein haploiden Stammes konnten jetzt höhere Mutationsraten als in früheren Versuchen ${ }^{7}$ erzielt werden.

Als Komplettmedium diente ein Hefeextrakt-Pepton-Glucose-Medium, als Minimalmedium eine Nährsalzlösung mit $1 \%$ Glucose und $100 \gamma$ Biotin/Liter. Zum Waschen wurde eine Salzlösung $(0,5 \% \mathrm{NaCl}$,
$0,012 \% \mathrm{MgSO}_{4}+7 \mathrm{H}_{2} \mathrm{O}$ ) benutzt. Alle Versuche wurden bei $30{ }^{\circ} \mathrm{C}$ durchgeführt. Weitere meihodische Einzelheiten sind in der früheren Arbeit ${ }^{7}$ aufgeführt bzw. werden unten bei den Versuchsbeschreibungen angegeben.

\section{Ergebnisse}

\section{Versuche mit verschiedenen Hemmstoffen}

Zunächst testeten wir in Modellversuchen eine Reihe von Antibiotika und anderen Stoffen, bei denen auf Grund von Angaben in der Literatur eventuell Anreicherungseffekte zu erwarten waren (Tab. 1). Über Versuche mit ${ }^{3} \mathrm{H}$-Thymidin und Penicillin wird in den darauf folgenden Abschnitten berichtet.

Für die Versuche wurden der Wildstamm und eine Tryptophan-Mangelmutante über Nacht in je $5 \mathrm{ml}$ Komplettmedium angezogen, 2-mal gewaschen und in $5 \mathrm{ml}$ Salzlösung suspendiert. Entweder 0,5 oder $0,05 \mathrm{ml}$ der Hefesuspension wurden in $5 \mathrm{ml}$ Minimalmedium mit $\mathrm{Zu}$ sätzen der zu prüfenden Substanz gegeben. Nach meist eintägiger Inkubation wurde auf Komplettagar plattiert, und die Abtötungsraten von Wildstamm und Mutanten wurden verglichen.

Von den untersuchten Stoffen (Tab. 1) hat keiner zu einer Anreicherung von Hefemutanten geführt, d. h. zu einer bevorzugten Abtötung des Wildstam-

\begin{tabular}{|c|c|c|c|c|}
\hline Verbindung & Herkunft & Lit. & Bemerkungen & Wirkung \\
\hline 4-Androsten-3.17-dion & $\begin{array}{l}\text { Fluka A.G. Chem. Fabrik } \\
\text { Buchs. }\end{array}$ & 9 & Steroid & ohne Befund \\
\hline „Moronal“ (Nystatin) & $\begin{array}{l}\text { Chem. Fabrik Von Heyden, } \\
\text { München }\end{array}$ & 6,10 & $\begin{array}{l}\text { Polyen-Antibiotikum aus } \\
\text { Streptomyces noursei }\end{array}$ & $\begin{array}{l}\text { Hemmung durch } \\
10-100 \gamma / \mathrm{ml}\end{array}$ \\
\hline,,$G M 83^{،}$ & Farbwerke Hoechst & - & Polyen-Antibiotikum & $\begin{array}{l}\text { Hemmung durch } \\
1 \gamma / \mathrm{ml}\end{array}$ \\
\hline Actidion (Cycloheximid) & $\begin{array}{l}\text { Upjohn Co., Kalamazoo, } \\
\text { USA. }\end{array}$ & 11 & $\begin{array}{l}\text { Antibiotikum aus Strept. } \\
\text { griseus }\end{array}$ & $\begin{array}{l}\text { Hemmung durch } \\
100 \gamma / \mathrm{ml}\end{array}$ \\
\hline Xanthocillin & $\begin{array}{l}\text { Chemie Grünenthal, } \\
\text { Stollberg, Rhld. }\end{array}$ & 12 & $\begin{array}{l}\text { Antibiotikum aus Penic. } \\
\text { notatum }\end{array}$ & ohne Befund \\
\hline Novobiocin & Farbwerke Hoechst & 13 & $\begin{array}{l}\text { Antibiotikum aus Strept. } \\
\text { niveus }\end{array}$ & ohne Befund \\
\hline Phyllomycin & $\begin{array}{l}\text { Dr. G. Schmidt-Kastner, } \\
\text { Bayer-Elberfeld }\end{array}$ & 14 & $\begin{array}{l}\text { Antibiotikum aus Strept. } \\
\text { umbrosus }\end{array}$ & $\begin{array}{l}\text { Hemmung durch } \\
1 \gamma / \mathrm{ml}\end{array}$ \\
\hline Bacimethrin & Synthese nach 15 & 16 & $\begin{array}{l}\text { Antibiotikum aus Bac. } \\
\text { megatherium }\end{array}$ & ohne Befund \\
\hline 8-Azaguanin & $\begin{array}{l}\text { Sigma, Chemical Co., } \\
\text { St. Louis, USA. }\end{array}$ & 17 & - & ohne Befund \\
\hline
\end{tabular}

Tab. 1. Wirkung verschiedener Verbindungen auf Saccharomyces cerevisiae.

\footnotetext{
8 F. Lingens u. O. Oltmanns, Naturwissenschaften 50, 232 [1963].

9 E. S. Maxwell, J. S. McGuire u. G. M. Tomkins, J. Bacteriol. 80, 1 [1960].

10 S. C. Kinsky, Proc. nat. Acad. Sci. USA 48, 1049 [1962].

11 K. Gundersen u. T. Wadstein, J. gen. Microbiol. 28, 325 [1962].

12 R. Beiersnorf u. W. Ahrens, Pharmacie 8, 796 [1953].
}

13 Tн. D. Brock, J. Bacteriol. 84, 779 [1962].

14 Patentschrift DBP Nr. 1061964/(1959).

15 H. C. Koppel, R. H. Springer, R. K. Robins u. C. C. Cheng, J. org. Chemistry 27, 1492 [1962].

16 F. Tanaka, S. Takeuchi, N. Tanaka, H. Yonehara, H. Umezawa u. Y. Sumiki, J. Antibiotics [Tokyo], Ser. A, 14, 161 [1961].

17 J. T. Wachsman u. R. Mangalo, J. Bacteriol. 83, 35 [1962]. 
mes. Dieses negative Resultat wird durch inzwischen von anderen Autoren veröffentlichte Befunde über die Wirkungsweise einzelner Antibiotika ${ }^{18}$ z. T. verständlich.

\section{Versuche mit Tritium-Thymidin}

Von Lubin ${ }^{19}$ ist eine weitere Methode zur Anreicherung von Bakterien-Mutanten beschrieben worden. Sie beruht auf der Aufnahme von TritiumThymidin durch in Minimalmedium wachsende Wildstammzellen und der Letalwirkung des TritiumZerfalles. Wegen der geringen Reichweite des vom Tritium emittierten $\beta$-Teilchens (maximal $6 \mu$ in Wasser) werden vermutlich nur die Zellen geschädigt, die Tritium inkorporiert haben, nicht aber benachbarte Zellen ${ }^{19}$. Wir haben diese Methode auch bei Hefen angewandt.

Im ersten Versuch wurden der Wildstamm und eine try ${ }^{-}$-Mutante $2 \mathrm{Stdn}$. in Minimalmedium mit ${ }^{3}$ H-Thymidin-Zusatz inkubiert. Die spezifische Aktivität des Thymidins betrug $1,9 \mathrm{C} / \mathrm{mMol}$, die Aktivität im Ansatz $40 \mu \mathrm{C} / \mathrm{ml}$. Nach der zweistündigen Inkubation wurden die Suspensionen im Eisschrank bei $+4{ }^{\circ} \mathrm{C}$ aufbewahrt. Von Zeit zu Zeit wurden Proben entnommen und die Überlebendraten ermittelt. Parallel zu dem Versuch mit Hefen wurde ein ganz entsprechender Versuch mit E.coli 15 und einer try-Mutante dieses Stammes durchgeführt.

In einem zweiten Versuch mit Hefen wurde der Wildstamm nach der Methode yon Moat, Peters und

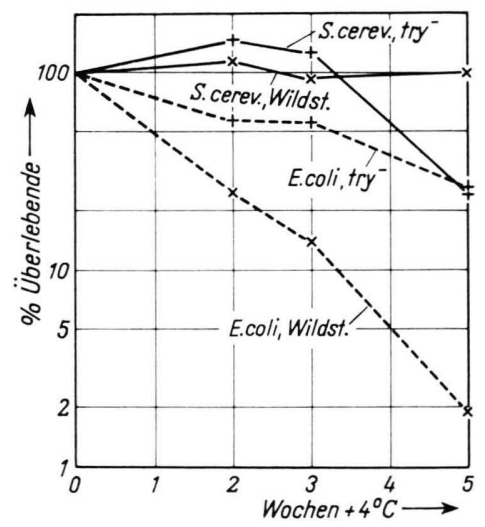

Abb. 1. Überlebendraten von Sacch. cerevisiae-Wildstamm und -Mutante und von E. coli-Wildstamm und -Mutante während einer mehrwöchigen Aufbewahrungszeit bei $+4{ }^{\circ} \mathrm{C}$ nach 2 -stdg. Inkubation mit ${ }^{3} \mathrm{H}$-Thymidin.

18 G. D. Shockman u. J. O. Lampen, J. Bacteriol. 84, 508 [1962].

19 M. Lubin, Science [Washington] 129, 838 [1959].

20 C. C. Gordon u. O. L. Stein, Radiat. Botany 2, 7 [1962].
$\mathrm{S}_{\mathrm{RB}}{ }^{6}$ mit einer rotgefärbten, Adenin-bedürftigen Mutante gemischt und die Mischpopulation wie oben mit Tritium-Thymidin inkubiert. In wöchentlichen Abständen wurden Proben entnommen und auf Komplettagar plattiert. Durch Auszählen der weißen und roten Kolonien lassen sich leicht Abtötungsraten von Wildstamm und Mutante und der Anreicherungseffekt ermitteln.

Die Ergebnisse des ersten Versuches sind in Abb. 1 dargestellt: Der Wildstamm von E. coli stirbt während der mehrwöchigen Aufbewahrungszeit bei $+4{ }^{\circ} \mathrm{C}$ bedeutend schneller ab als die Mutante. Bei Saccharomyces beobachtet man dieses Phänomen nicht. - Im zweiten Versuch sank die Zahl der überlebenden Hefezellen im Zeitraum von 8 Wochen auf etwa die Hälfte des Ausgangswertes, jedoch bei dem Wildstamm und der $\mathrm{ad}^{-}$-Mutante in gleicher Weise.

Da bei Bakterien mit der Tritium-Thymidin-Methode Mutanten angereichert werden konnten ${ }^{19}$ und auch in dem von uns durchgeführten Vergleichsversuch (Abb. 1) der Wildstamm von E. coli mehr geschädigt wurde als die Mutante, stellt sich die Frage, warum ein gleicher Effekt bei Hefen nicht zu erzielen ist. Eine Erklärung dafür könnte darin liegen, daß Hefen wie andere Pilze ${ }^{20}$ Thymin und Thymidin schlecht aufnehmen bzw. verwerten ${ }^{21}$. Es wäre in diesem Falle angebracht, statt ${ }^{3} \mathrm{H}$-Thymidin z. B. ${ }^{3}$ H-Leucin zu verwenden, das von Lubin ${ }^{22}$ ebenfalls mit Erfolg zur Anreicherung von E.coli-Mutanten benutzt wurde. Wir haben derartige Versuche nicht durchgeführt, weil wir inzwischen in Vorversuchen mit Penicillin aussichtsreichere Ergebnisse erhalten hatten ${ }^{8}$.

\section{Versuche mit Penicillin}

In einer früheren Arbeit ${ }^{8}$ konnten wir den Befund von Strutz und Kuntze ${ }^{23}$ bestätigen, daß hohe Penicillin-Konzentrationen Saccharomyces cerevisiae abtöten. Wir hatten ferner gewisse Anreicherungseffekte nachgewiesen: Eine in Modellversuchen eingesetzte Tryptophan-Mangelmutante und eine Adenin ${ }^{-}$-Mutante wurden durch Penicillin langsamer abgetötet als der Wildstamm ${ }^{8}$.

Im folgenden Experiment wird untersucht, welchen Einfluß die Penicillin-Konzentration auf die Abtötungsraten hat.

21 D. H. W Wlitamson, Biochem. J. 90, 25 P [1964].

22 M. Lubin, J. Bacteriol. 83, 696 [1962].

23 I. Strutz u. C. Kuntze, Naturwissenschaften 42, 464 [1955]. 

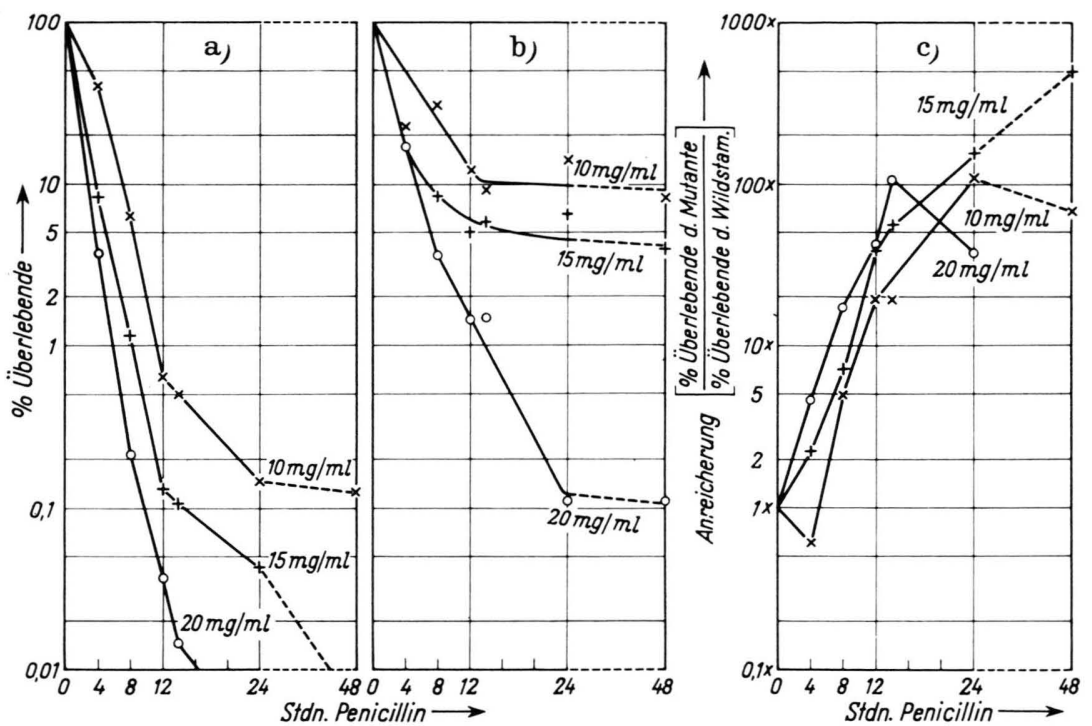

Abb. 2. Abtötung von Saccharomyces cerevisiae-Wildstamm (a) und ad-'Mutante (b) durch Penicillin-G-Kalium-Lösung verschiedener Konzentration und (c) Verhältnis der Uberlebendraten von Mutante und Wildstamm („Anreicherung“).

Der Wildstamm und die rote Adenin-Mutante E 188 wurden 2 Tage auf Komplettagar angezogen. Je zwei große Platinösen voll Hefezellen wurden in je $5 \mathrm{ml}$ Salzlösung suspendiert, 2-mal gewaschen, in $5 \mathrm{ml} \mathrm{Salz}$ lösung resuspendiert und im Verhältnis $30: 1$ (Wildstamm : Mutante) gemischt. Je $0,05 \mathrm{ml}$ dieser Mischsuspension wurden in $350-\mathrm{ml}-\mathrm{Kolben}$ mit $5 \mathrm{ml}$ Minimalmedium gegeben, so daß eine Zellkonzentration von ca. $10^{6}$ Zellen $/ \mathrm{ml}$ resultierte. Die 3 Kolben wurden mit 50, 75 bzw. $100 \mathrm{mg}$ Penicillin-G-Kalium versetzt (entsprechend 10, 15 bzw. $20 \mathrm{mg} / \mathrm{ml}$ ) und in einem Wasserbad bei $30{ }^{\circ} \mathrm{C}$ inkubiert. $\mathrm{Zu}$ verschiedenen $\mathrm{Zei}$ ten wurden Proben entnommen, auf Komplettagar plattiert und die Überlebendraten von Wildstamm und Mutante festgestellt.

Abb. 2 bringt die Ergebnisse: Nicht nur die Abtötungsrate des Wildstammes (Abb. $2 \mathrm{a}$ ), sondern auch die der Mutante (Abb. 2 b) nimmt mit der Penicillin-Konzentration zu. Für jede Konzentration scheint es einen bestimmten Prozentsatz von überlebenden Mutantenzellen zu geben, er liegt für die angegebenen Konzentrationen bei 10\%, 4\% bzw. 0,1 Prozent. Der Anreicherungseffekt (\% überlebende Zellen der $\mathrm{ad}^{-}$-Mutante: \% überlebende Wildstammzellen, Abb. 2 c) ist bei einer Konzentration von $15 \mathrm{mg}$ Penicillin/ml nach $48 \mathrm{Stdn}$. Inkubationsdauer am größten (490-fache Anreicherung, Abb. 2 c). Diese Konzentration von $15 \mathrm{mg} / \mathrm{ml}$ wählten wir für die weiteren Versuche, denn bei einer Konzentration von $10 \mathrm{mg} / \mathrm{ml}$ werden zu wenig Wildstammzellen abgetötet, bei $20 \mathrm{mg} / \mathrm{ml}$ zuviel Mutantenzellen.
Es war von Interesse zu sehen, ob und wie stark andere Hefe-Mutanten durch Penicillin abgetötet würden. Im nächsten Versuch wurden daher nach der oben angegebenen Methode mehrere Sacch. cerevisiae-Mutanten mit $15 \mathrm{mg}$ Penicillin/ml behandelt (Abb. 3). Von 2 Tryptophan-Mutanten war die eine (E 40) unempfindlicher gegen Penicillin als der Wildstamm, die andere (HK 145) wurde ebenso wie eine Lysin-bedürftige Mutante (G 47) sogar noch schneller als der Wildstamm abgetötet. Eine weitere Mutante (KP 400, mit Adenin- und Argininbedarf) war relativ resistent gegen Penicillin. Das konnte in diesem Fall erwartet werden, denn diese Mutante war in einem Anreicherungsversuch nach 48-stdg. Penicillin-Einwirkung isoliert worden (s. u.). - Zum Vergleich haben wir einen Versuch mit E. coli durchgeführt (Abb. 4) : Die TryptophanMutante wurde durch $15 \mathrm{mg}$ Penicillin/ml nur bis auf $50 \%$ abgetötet, und sie starb erst ab, nachdem sie durch eine Gabe von Tryptophan zum Wachsen gebracht worden war.

Die bisher geschilderten Versuche zeigen, daß in Minimalmedium mit $15 \mathrm{mg}$ Penicillin $/ \mathrm{ml}$ relativ gute Anreicherungseffekte zu erzielen sind. Bei dieser Konzentration bleiben jedoch nicht alle Hefemutanten am Leben. Die Überlebendrate der Mutanten scheint nicht von ihrem Nährstoffbedarf abhängig zu sein, wie die verschieden große Empfindlichkeit der beiden Tryptophanmutanten zeigt. 
Anreicherung Methansulfonsäureäthylester-induzierter Mutanten

Bei der Penicillin-Anreicherung von Mutanten werden meistens mehrere Schritte ausgeführt ${ }^{24}$ :

1. Induktion von Mutanten durch ein mutagenes Agens, 2. Inkubation der behandelten Zellen in Komplettmedium zur genotypischen Ausprägung

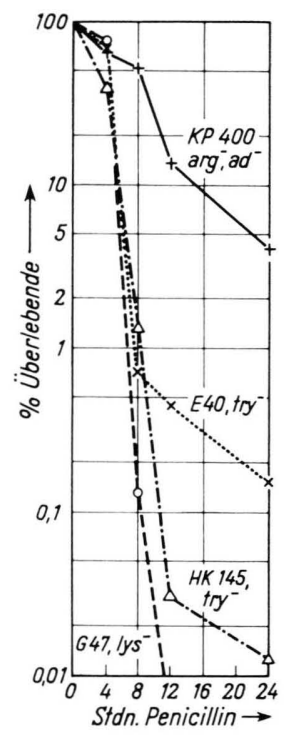

Abb. 3. Abtötung von Saccharomyces cerevisiae-Mutanten durch $15 \mathrm{mg}$ Penicillin $/ \mathrm{ml}$.

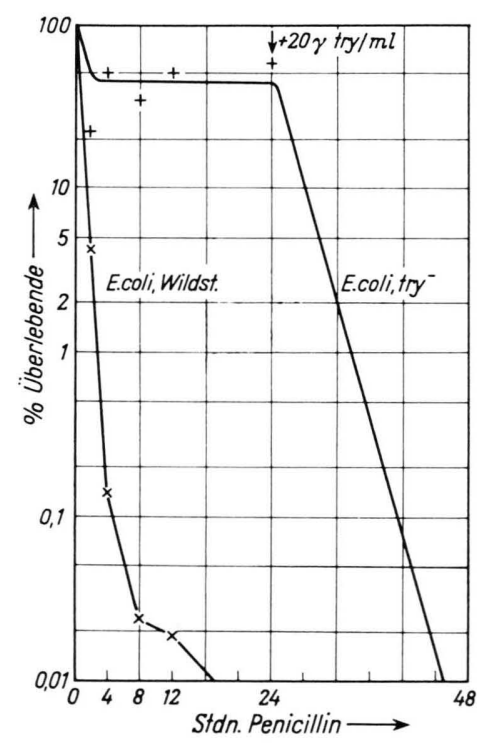

Abb. 4. Abtötung von E. coli-Wildstamm und try ${ }^{-}$-Mutante durch $15 \mathrm{mg}$ Penicillin/ml. Nach $24 \mathrm{Stdn}$. Inkubation in Minimalmedium wurden $20 \gamma$ l-Tryptophan/ml zugegeben.

24 B. D. Davis, Experientia [Basel] 6, 41 [1950]. der Mutationen, 3. Inkubation in N-freiem Minimalmedium zur phänotypischen Ausprägung, 4. Behandlung der Population in Minimalmedium mit Penicillin, 5. Isolierung der Mutanten.

In unseren Versuchen mit Hefen konnten wir keinen besonderen Vorteil der Aushungerung in Nfreiem Minimalmedium bemerken. Nach einigen Vorversuchen benutzten wir schließlich folgende Versuchsanordnung zur Anreicherung induzierter Hefemutanten:

Der Wildstamm S $288 \mathrm{C}$ wurde 2 Tage lang auf Komplettagar vorgezüchtet. Dann wurden mit einer Platinöse Hefezellen entnommen, in $5 \mathrm{ml}$ Salzlösung suspendiert, 2-mal gewaschen und in $5 \mathrm{ml} \mathrm{Salz-}$ lösung aufgeschwemmt. Die Zellen von $2,5 \mathrm{ml}$ dieser Suspension wurden mit einer 2-proz. Lösung von Methansulfonsäureäthylester-MS $\ddot{A}-(\mathrm{Fa}$. Th. Schuchardt, München) in Phosphatpuffer bei $p_{\mathrm{H}} 7,3-7,0$ im Wasserbad unter Schütteln inkubiert. Nach 2 Stdn. (Versuch 1) bzw. 1,5 Stdn. (Versuch 2) Inkubation (ca. 10\% Überlebende) wurden Proben 1: 10 verdünnt und auf Komplettagar plattiert. Es wuchsen in 3 Tagen ca. 15000 Kolonien je Platte. Die Zellen wurden mit Salzlösung von einer Platte abgeschwemmt, 2-mal gewaschen und auf eine Konzentration von $10^{8}$ Zellen $/ \mathrm{ml}$ verdünnt. $0,05 \mathrm{ml}$ dieser Suspension wurden mit $5 \mathrm{ml}$ Minimalmedium und $75 \mathrm{mg}$ Penicillin-G-Kalium im Wasserbad unter Schütteln inkubiert. $\mathrm{Zu}$ verschiedenen Zeiten wurden Proben entnommen und nach der früher angegebenen Methode ${ }^{7}$ die Mutanten isoliert und identifiziert.

Aus Tab. 2 ist das Ergebnis zweier Versuche ersichtlich: Nach der Methansulfonsäureäthylester-Behandlung erhält man bei Überlebendraten zwischen 6,5 und $49 \%$ ungefähr $5-15 \%$ Mutanten unter den Überlebenden. Die 3-tägige Inkubation der MSÄbehandelten Zellen auf Komplettagar vermindert im Versuch 2 die Mutationsrate von 17 auf 9\%, was darauf hindeutet, daß sich die Wildstammzellen auf der Komplettagarplatte etwas stärker vermehrt haben als die mutierten Zellen.

Die Behandlung mit Penicillin führte zu einer Zunahme des Mutantenanteiles von 12 auf 50\% im 1. Versuch und von 9 auf $20 \%$ im 2. Versuch. Die Mutantenausbeute läßt sich also durch die Penicillinbehandlung merklich steigern.

Wie die Untersuchung des Nährstoffbedarfes der isolierten Mutanten zeigt (Tab. 2), treten nach der Penicillin-Anreicherung kaum weniger Mutantentypen auf als in Versuchen ohne Penicillin-Einwirkung. Auffallend ist dagegen der große Anteil Adenin-bedürftiger Mutanten, die im 1. Versuch $71 \%$, im 2. Versuch $22 \%$ aller Mutanten ausmachen. 


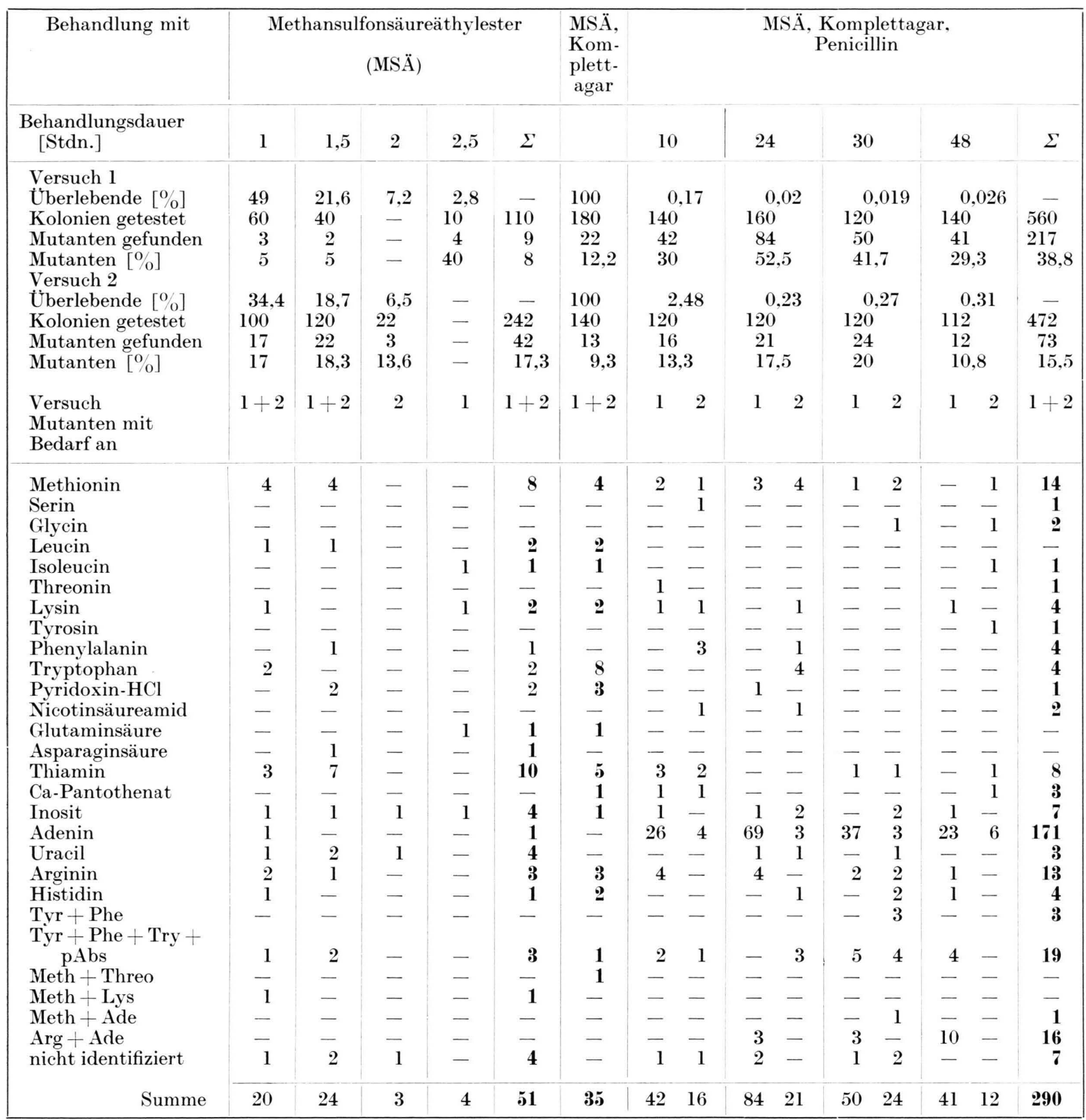

Tab. 2. Anzahl und Art der vor und nach Penicillineinwirkung isolierten Mutanten.

\section{Diskussion}

Zur Anreicherung von Mangelmutanten. - Von allen untersuchten Agenzien hatte nur Penicillin die Eigenschaft, biochemische Mangelmutanten von Saccharomyces cerevisiae anzureichern. Die Penicillinbehandlung vermehrte den Anteil der Methansulfonsäureäthylester-induzierten Mutanten in unseren
Versuchen auf das 2-4-fache. Diese Erhöhung des Mutantenanteiles ist nicht besonders groß, selbst wenn man bedenkt, daß wegen der hohen Mutationsrate bei Verwendung von MSÄ nur noch eine etwa 10-fache Erhöhung des Mutantenanteiles möglich ist.

Nach der Penicillin-Anreicherung wurden relativ viele Adenin-bedürftige Mutanten gefunden. Eine 
gleichmäßigere Verteilung der Mutantentypen ist im allgemeinen erwünschter, denn oft wird nicht gerade der häufig auftretende Typ untersucht werden. Es kommt hinzu, daß die zahlreichen Mutanten eines Typs nicht unbedingt unabhängig voneinander entstanden sein müssen. Die durch ein mutagenes Agens induzierten Mutanten werden in Anreicherungsversuchen zur genotypischen Ausprägung des Merkmales in Komplettmedium vermehrt. Daher können Zellen mit gleichem genetischen Defekt aus einem Klon stammen und mehrmals isoliert werden. Adelberg und Myers ${ }^{25}$ haben aus diesem Grunde eine Modifikation der Penicillin-Technik entwickelt, bei der die Anreicherungsprozedur auf festen Nährböden durchgeführt wird. Mit dieser Technik werden Mutanten eines Klones nur einmal isoliert. Sie ist jedoch mit einigem Arbeitsaufwand verbunden, und Verluste an Mutanten sind nicht auszuschließen.

In welchem Ausmaß in unseren Versuchen Mehrfachisolierungen von Mutanten eine Rolle spielen, läßt sich nur abschätzen. Auf der einen Seite kommen Einfach-Isolierungen sicher häufiger vor als die Tabelle erkennen läßt (z. B. unterscheiden sich die vier Tryptophan-Mutanten und die zwei Nicotinsäure-Mutanten in Wachstumstests mit den entsprechenden Vorstufen). Andererseits gehen die $16 \mathrm{Mu}$ tanten mit Arginin- und Adenin-Bedarf wohl auf eine Zelle zurück, denn Mutanten dieses Typs haben wir vorher weder in eigenen Versuchen gefunden noch wurden sie in der Literatur beschrieben.

Da biochemische Zusammenhänge zwischen der Arginin- und der Adenin-Biosynthese nicht bestehen, liegt bei den $\mathrm{arg}^{-}-, \mathrm{ad}^{-}$-Mutanten eventuell sogar der seltene Fall einer gleichzeitigen Induktion von zwei Mutationen in einer Zelle vor. Eine mehrmalige Induktion solcher Mutanten in einem Versuch wäre äußerst unwahrscheinlich.

Die zahlreichen Adenin-Mutanten haben sicher nicht nur in einer einzigen Mutation ihren Ursprung, denn im Versuch 1 wurden unter ihnen zwei rote Mutanten gefunden, im Versuch 2 eine rote Mutante. Wegen der großen Häufigkeit dieser Mutanten liegt jedoch trotzdem der Verdacht nahe, daß ein Teil davon Mehrfachisolierungen zuzuschreiben ist.

\footnotetext{
25 E. A. Adelberg u. J. W. Myers, J. Bacteriol. 65, 348 [1953].

* Versuche an Hefen mit diesem Agens: F. Lingens u. O. Oltman, Z. Naturforschg., in Vorbereitung.
}

Das gehäufte Auftreten von Mutanten eines Typs und die Möglichkeit von mehrmaligen Isolierungen von mutierten Zellen eines Klones mindern den praktischen Wert des hier beschriebenen Anreicherungsverfahrens erheblich. Man könnte nun versuchen, die modifizierte Technik von Adelberg und Myers ${ }^{25}$ auch bei den Hefen anzuwenden. Wir halten es aber für besser, auf Anreicherungsverfahren ganz zu verzichten, da man durch die Anwendung hochwirksamer Mutagene, wie z. B. Methansulfonsäureäthylester oder auch 1-Nitroso-3-nitro-1-methylguanidin *, leicht Mutationsraten von über $10 \%$ erreichen kann. Die Penicillinmethode und andere Anreicherungsverfahren mögen eine gewisse Bedeutung haben in Fällen, in denen es darauf ankommt, Mutanten nach Induktion mit wenig wirksamen Mutagenen zu isolieren.

Zur Wirkung des Penicillins auf Saccharomyces cerevisiae. Penicillin greift in die Biosynthese der Bakterienzellwand ein, wie Untersuchungen verschiedener Autoren gezeigt haben. Nach Versuchen an grampositiven Bakterien nahm man an, daß der Einbau des Muropeptides in die Zellwand durch Penicillineinwirkung unterbunden und dieses daher in Form eines Uridinnucleotides akkumuliert würde. Experimente mit gramnegativen Organismen ließen jedoch Zweifel an der allgemeinen Gültigkeit dieser speziellen Vorstellungen aufkommen ${ }^{26,27}$.

Die Zellwand der Hefe ist nach den bisherigen Vorstellungen ${ }^{28}$ ganz anders aufgebaut als die der Bakterien, insbesondere ist keine Muropeptidstruktur gefunden worden. Da Penicillin trotzdem abtötend auf Hefen wirkt, müssen entweder die ursprünglichen Vorstellungen vom Penicillin-Wirkungsmechanismus (Verhinderung des Muropeptideinbaues) oder die von der Zusammensetzung der Hefezellwand ergänzt werden.

Es soll kurz diskutiert werden, ob nicht das Penicillin selbst, sondern eine Verunreinigung des Penicillins die Wirkung auf Hefen hervorrufen könnte. Zwei Beobachtungen sprechen gegen eine solche Verunreinigung: Erstens haben wir Penicillin verschiedener Chargen, nämlich Penicillin als Kaliumund als Natriumsalz verwendet. Die hypothetische Verunreinigung müßte also ganz allgemein in jedem Penicillinpräparat enthalten sein und übrigens auch in dem Penicillin, das Strutz und Kuntze 1955 ver-

\footnotetext{
26 R. Plapp u. O. Kandler, Arch. Mikrobiol. 50, 171 [1965].

27 R. Plapp u. O. Kandler, Arch. Mikrobiol. 50, 282 [1965].

28 W. J. Nickerson, Bacteriol. Rev. 27, ${ }_{3} 05$ [1963].
} 
wendet haben. Zweitens wird interessanterweise eine E. coli-Mutante in Minimalmedium, in dem sie nicht wachsen kann, auch durch die von uns verwendeten hohen Penicillin-Konzentrationen nicht abgetötet. Die im Penicillin enthaltene hypothetische Verunreinigung müßte also für Saccharomyces cerevisiae giftig sein, nicht aber für E. coli. Wir halten die Existenz einer sochen Verunreinigung deshalb für unwahrscheinlich.

Im Gegensatz zu den Verhältnissen bei Bakterien wirkt Penicillin in Minimalmedium nicht nur auf den Hefewildstamm abtötend, sondern auch auf die Mutanten, die nicht wachsen. Das würde gegen einen Wirkungsmechanismus sprechen, der in den Zellwand-Aufbau-Mechanismus eingreift. Jedoch ist es durchaus vorstellbar, daß die Hefezellwand auch in nicht wachsenden Zellen ständig auf- und abgebaut würde und dabei dem Angriff des Penicillins ausgesetzt wäre. Die Empfindlichkeit der Mutanten gegen Penicillin reicht demnach nicht aus als Beweis für eine andersartige Wirkung des Penicillins bei Hefen.

Die Synthese der Bakterienzellwand läßt sich nicht nur durch Penicillin, sondern auch durch Novobiocin und D-Cycloserin hemmen. Novobiocin hatte in unseren Versuchen keinen Effekt auf Saccharomyces. Da Novobiocin im sauren Hefe-Minimalmedium nur schlecht löslich ist, lassen sich aus seiner Unwirksamkeit keine weitreichenden Schlüsse ziehen. Das gut lösliche D-Cycloserin wirkte in einem kürzlich angestellten Experiment in hohen Konzentra-

29 A. E. Braunstein, R. M. Azarkh u. Hsu Ting Seng, Biochimija 26, 882 [1961] ; zit. n. F. C. Neuhaus u. J. L. Lynch, Biochem. biophysic. Res. Commun. 8, 377 [1962]. tionen abtötend, jedoch sind von diesem Antibiotikum neben dem Eingreifen in den Zellwand-Aufbaumechanismus noch andere Wirkungsmechanismen bekannt, z. B. die Hemmung von Transaminasen ${ }^{29,} 30$.

Bei Bakterien führt die Einwirkung von Penicillin auf wachsende Zellen zur Auflösung der Zellwand und zur Lyse. Die durch Penicillin abgetöteten Hefe-Zellen unterscheiden sich im mikroskopischen Bild nicht von lebenden Zellen. Auch diese Beobachtung schließt noch nicht eine Hemmung des Zellwand-Aufbaumechanismus durch Penicillin aus: Wenn nur eine der Schichten der Hefezellwand gegen Penicillin empfindlich wäre, könnten doch die anderen Schichten ausreichen, um der Penicillinbehandelten Zelle die Form zu erhalten.

Die Frage, ob das Penicillin bei Hefen ebenso wie bei Bakterien in den Zellwand-Aufbaumechanismus eingreift, kann also auf Grund der hier geschilderten Versuche nicht entschieden werden. Muraminsäure ließ sich aus Hefezellwänden nicht isolieren ${ }^{31}$.

Fräulein G. Tribbensee und Fräulein U. Schuster danken wir für fleißige und sorgfältige Mitarbeit. Dem Bundesministerium für wissenschaft liche Forschung und dem Fonds der Che mischen Indust rie danken wir für Unterstützung dieser Arbeit. Von der B a y e r A G, W.-Elberfeld, erhielten wir dankenswerterweise eine größere Menge Penicillin und ein weiteres Antibioticum, von den $\mathrm{F}$ a r b w e r k e n $\mathrm{H}$ o e ch st, Frankfurt, und der Chemie Grünenthal, Stollberg, verschiedene Antibiotika.

30 P. Oенме, Naturwissenschaften 50, 20 [1963].

31 F. Lingens u. J. Daum, unveröffentlicht. 